\title{
Hippocampal Damage Associated with Prolonged Glucocorticoid Exposure in Primates
}

\author{
Robert M. Sapolsky, ${ }^{1}$ Hideo Uno, ${ }^{2}$ Charles S. Rebert, ${ }^{3}$ and Caleb E. Finch ${ }^{4}$ \\ 'Department of Biological Sciences, Stanford University, Stanford, California 94305, ${ }^{2}$ Regional Primate Research Center, \\ The University of Wisconsin, Madison, Wisconsin 53715, ${ }^{3} \mathrm{SRI}$ International, Menlo Park, California 94025, and ${ }^{4}$ Andrus \\ Gerontology Center and Department of Biological Sciences, University of Southern California, Los Angeles, \\ California 90089
}

In the laboratory rat and guinea pig, glucocorticoids (GCs), the adrenal steroids that are secreted during stress, can damage the hippocampus and exacerbate the hippocampal damage induced by various neurological insults. An open question is whether GCs have similar deleterious effects in the primate hippocampus. In fact, we showed that sustained and fatal stress was associated with preferential hippocampal damage in the vervet monkey; however, it was not possible to determine whether the excessive GC secretion that accompanied such stress was the damaging agent. The present study examines this possibility. Pellets of cortisol (the principal GC of primates) were stereotaxically implanted into hippocampi of 4 vervet monkeys; contralateral hippocampi were implanted with cholesterol pellets as a control. One year later at postmortem, preferential damage occurred in the cortisol-implanted side. In the cholesterol side, mild cell layer irregularity was noted in 2 of 4 cases. By contrast in the cortisol-exposed hippocampi, all cases had at least 2 of the following neuropathologic markers: cell layer irregularity, dendritic atrophy, soma shrinkage and condensation, or nuclear pyknosis. Damage was severe in some cases, and was restricted to the CA3/CA2 cellfield. This anatomical distribution of damage, and the cellular features of the damage agree with that observed in instances of GC-induced toxicity in the rodent hippocampus, and of stress-induced toxicity in the primate hippocampus. These observations suggest that sustained GC exposure (whether due to stress, Cushings syndrome or exogenous administration) might damage the human hippocampus.

The hippocampus is a principal neural target for glucocorticoids (GCs), the adrenal steroids secreted during stress. That structure contains high concentrations of corticosteroid receptors and has a marked sensitivity to the hormones, as marked by neurochemical and electrophysiological changes (McEwen et al., 1986). Growing evidence shows that prolonged, supraphysiological elevations of GCs can damage the hippocampus in a direction which appears to accelerate aging. Injections of GCs damage

Received Dec. 4, 1989; revised Apr. 6, 1990; accepted Apr. 6, 1990.

Support for R.M.S. was provided by NIH grant AG06633 and the Alzheimer's Disease and Related Disorders Association. Support for C.E.F. was provided by NIH grant AGO7909 and from the John D. and Catherine T. MacArthur Foundation.

Correspondence should be addressed to Robert Sapolsky at the above address. Copyright $@ 1990$ Society for Neuroscience 0270-6474/90/092897-06\$03.00/0 pyramidal neurons of guinea pigs (Aus der Muhlen and Ockenfels, 1969) and rats (Sapolsky et al., 1985). Moreover, chronic stress causes premature aging changes in hippocampal electrical activitics (Kcrr ct al., 1986) and, when it occurs prenatally, causes a reduction in pyramidal neuron number and retarded development of dendritic arborization (Uno et al., 1989a). Conversely, reducing exposure to GCs by behavioral (Meaney et al., 1988) or surgical means (adrenalectomy; Landfield et al., 1981) protects the hippocampus from senescent neuron loss. Finally, GCs, in concentrations that are not themselves toxic, impair the ability of hippocampal neurons to survive various neurological insults, including hypoxia-ischemia, excitotoxic seizures, and exposure to antimetabolites (Sapolsky, 1985, 1986; Sapolsky and Pulsinelli, 1985; Theoret et al., 1985; Koide et al., 1986; Morse and Davis, 1989).

These findings, all derived from rodent studies, suggest that prolonged exposure to GCs or to stress can accelerate hippocampal neuron loss during aging, as well as increase the severity of neurologic insults to the hippocampus. It is important to determine whether these observations apply to the primate and/ or human hippocampus. Recently, we studied vervet monkeys who died spontaneously after a period of sustained social stress. Along with pathologies that included multiple gastric ulcers, hyperplastic adrenals, entero-colitis and splenic lymphoid depletion, the animals had marked and preferential hippocampal degeneration with an anatomical distribution and cellular pattern very similar to the neuron loss in the rat hippocampus after sustained GC exposure (Uno et al., 1989b). This suggested that prolonged stress could damage the primate hippocampus. However, those data did not make it possible to determine whether the degeneration was, in fact, caused by GCs. The present study examines this possibility.

\section{Materials and Methods}

Subjects. Four young adult (5-8 years old) male vervet monkeys ( $C$. aethiops) purchased from Hahneman Medical College, Philadelphia, PA. The monkeys were housed individually in primate cages $\left(0.4 \mathrm{~m}^{2}\right.$ floor area, $0.8 \mathrm{~m}$ high) with food (Purina regular monkey chow) provided twice daily; water was available ad libitum. Before the experiment was started, the monkeys were quarantined for $30 \mathrm{~d}$, during which time their general health status was determined and T.B. tests were carried out. Room temperature was maintained at $22 \pm 1^{\circ} \mathrm{C}$; humidity was $40-70 \%$. The facilities at SRI International are fully accredited by the AALAC and all procedures were reviewed and approved by SRI's Animal Care Committee.

Surgical implantation of steroid-secreting pellets. The general strategy was to surgically implant pellets secreting either cortisol (the species- 


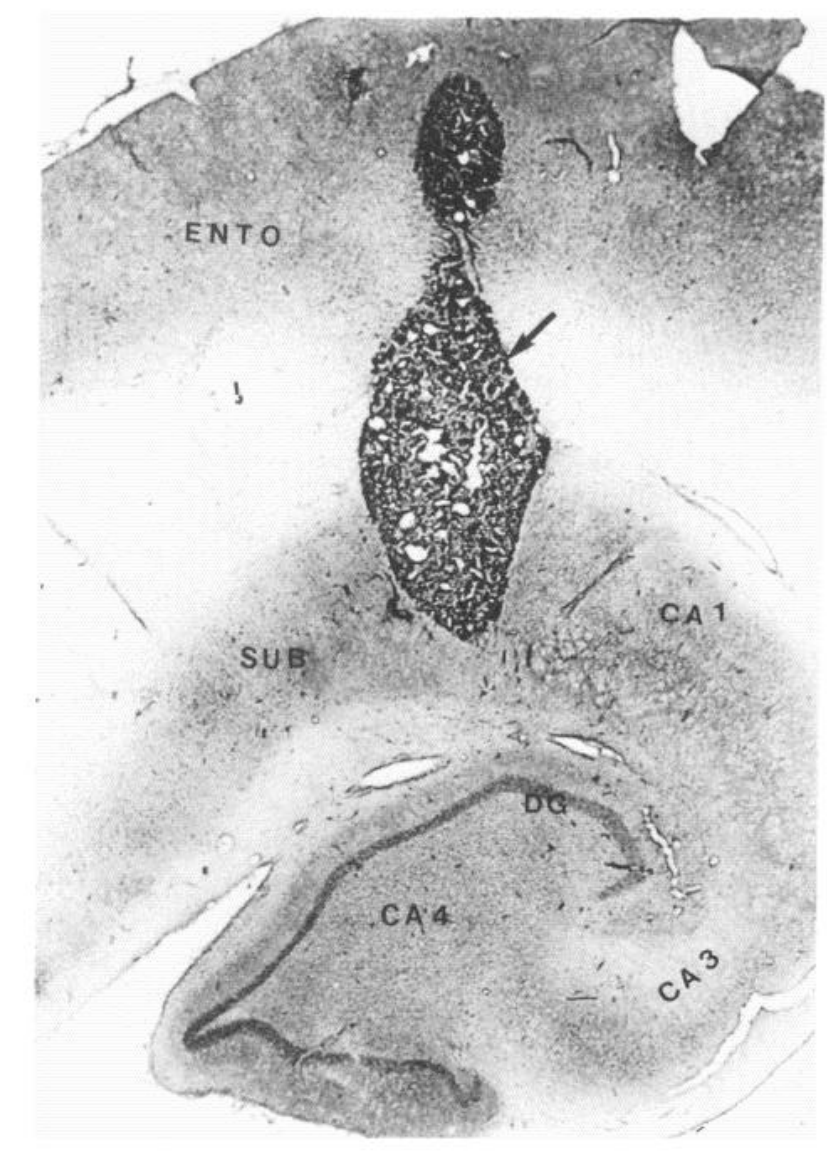

Figure 1. A pellet (arrow) located in the CA1 region through the entorhinal gyrus. $D G$, dentate gyrus. CA1, 3, 4 and sub: subiculum.

typical GC of primates) or cholesterol into the hippocampus. The latter was chosen as a control since it is the precursor to all steroid hormones, without having any hormonal effects itself; thus, it controls for any nonreceptor mediated effects. GCs were implanted locally, rather than administered systemically, to avoid the vast number of pathologic consequences of peripheral overexposure to GCs.

Pellets containing cortisol or cholesterol, purchased from Innovative Research of America (Gaitharsburg, MD) were pulverized and reconfigured to be approximately $1 \times 3 \mathrm{~mm}$ rods (a diameter such that the rods fit snugly into an 18 gauge biopsy needle). These were stereotaxically implanted into the hippocampi. Two cortisol pellets were implanted into one hippocampus, and 2 cholesterol into the other; sides were alternated from monkey to monkey. The 2 pellets on each side were separated by $5 \mathrm{~mm}$ in the anterior-posterior plane. Intended locations were 5 and $10 \mathrm{~mm}$ posterior to, and $10-11 \mathrm{~mm}$ below, the anterior commissure; and 10-13.5 mm lateral to midline, depending on the monkey and anterior or posterior location.

All surgical procedures were carried out aseptically. In preparation for surgery, the monkeys were lightly anesthetized with ketamine hydrochloride $(15 \mathrm{mg} / \mathrm{kg}$ i.m.). Atropine sulfate $(0.05 \mathrm{mg} / \mathrm{kg}$ i.m.) was administered to inhibit mucous membrane secretions. Calves were shaved and a cannula was inserted into one saphenous vein. Sodium pentobarbital was administered via the cannula to effect deep anesthesia. Two surgical procedures were carried out. First, the methods described by Rebert (1988) for coordinating stereotaxic coordinates with proton magnetic resonance images of the brain was used to develop stereotaxic coordinates. This involved placing copper sulfate-filled glass beads in the skull parallel to the Horsely-Clark plane and using one of the beads, visible in the sagittal image, as a reference point. Placements were calculated from measurements made on film negatives. Images were made by Dr. Ralph Hurd (General Electric, Fremont, CA). The monkeys were anesthetized with ketamine and sodium pentobarbital (via saphenous catheter) before transport to the imaging facility and maintained in moderate anesthesia in the magnet by periodic infusions of pentobarbital, using respiratory rate as a guide.
The second surgical procedure, carried out 34 or $36 \mathrm{~d}$ after the first surgery and 2-13 d after scanning, consisted of implanting the cortisol and cholesterol rods. Standard, aseptic stereotaxic methods were employed, except the top center of the posterior glass bead previously implanted in the skull was used as stereotaxic zero. Once the tip of the biopsy needle was lowered to $1.5 \mathrm{~mm}$ above the desired depth, the needle was raised $1.5 \mathrm{~mm}$ and the rod was pushed free of the needle.

Monkeys were examined daily by veterinarians skilled in primate care; no postsurgical abnormalities were observed in behavior, eating and drinking, weight, or urinary or bowel functions. At no point did animals show any evidence of Cushingoid symptoms (e.g., fat gain and redistribution causing moon facies and buffalo hump, muscle wastage, thinning and ulceration of skin).

One year after implantation of the steroid-secreting pellets, the monkeys were killed. (This interval was the longest period of time that funding allowed for housing of animals.) There was no information available as to the rate that pellets would secrete steroid when implanted in primate brain tissue. It was not possible to determine how much steroid remained in each pellet at the time of death (as removing the pellets would distort the tissue to be used for neuropathological analysis), and it was considered unacceptable to kill a separate group of monkeys after a year merely to quantify the extent of steroid release. Thus, the total amount and pattern of steroid exposure over the year was not known.

Animals were deeply anesthetized with ketamine and pentobarbital and perfused intracardially. After chest opening, $0.1 \mathrm{ml} / \mathrm{kg}$ heparin $(10$ units $/ \mathrm{ml}$ ) was injected into the right ventricle. This was followed by infusion of $300 \mathrm{ml}$ Ringer's solution and 2.0 liters of fixative (4\% paraformaldehyde in $0.1 \mathrm{M}$ phosphate buffer; $\mathrm{pH}=7.4$ ).

Neuropathological analysis. All analyses were done without knowledge as to which hemisphere received the cortisol or the cholesterol pellet. Brains were fixed by perfusion as described above and stored in the same fixative. Coronal sections, cut parallel to the needle tracks in the bilateral parietal lobes, were prepared by frozen sectioning $(50 \mu \mathrm{m}$ thick) and stained with cresyl violet. Other hippocampal sections were cut coronally $1 \mathrm{~mm}$ caudal from the uncus, cut vertically to the cortical surface, and were embedded in glycol methacrylate resin (JB4 embedding kit, Polysciences, Inc.). Pre- and post-central gyri of the cerebral cortex were cut and prepared for microscopic sectioning in a manner similar to the hippocampal sections. Plastic sections ( $3 \mu \mathrm{m}$ thick) were stained with cresyl violet and impregnated with silver by the LesterKing method.

Quantitative analysis of hippocampal neurons was performed in 3 plastic sections chosen from semi-serial sections in each case; the anatomical subdivisions refer to those previously specified in the brain of the Old World monkey (Rakic and Nowakowski, 1981; Uno et al., 1983, 1989). Neuropathological changes of neuron were manifested as a shrinkage or dark staining of the perikaryon, nucleus and dendritic processes, as previously defined (Sumi et al., 1984; Uno et al., 1989a, 1990).

Overall hippocampal dimensions were determined by measuring the anterior/posterior extent of the entire structure, and the dorsal/ventral and medial/lateral lengths of the structure in the 3 plastic-embedded sections used for the quantitative analysis.

Statistical analysis. Data derived from cell counting were analyzed by 2 -way ANOVA and presented as mean \pm SEM. The area was measured with an IPM PC using the trapezoidal method.

\section{Results}

Accuracy of pellet implantation. Grossly, 2 small indented holes were found in the bilateral pre- and post-central gyri of each brain approximately $12.0 \mathrm{~mm}$ lateral from the mid-sagittal line. The cerebral tissue around the needle tracks and cylindrical pellets showed no hemorrhagic or degenerative changes. In 3 of 4 brains, pellets were located in the Cornus Ammon (CA1) through the parahippocampal gyrus (Fig. 1). In one hippocampal section, a pellet perforated the dentate gyrus and was located in the hilus of the gyrus (brain number 1).

Neuropathological findings. The overall size of the hippocampus (dorsal/ventral, medial/lateral and anterior/posterior extents) did not differ according to treatment (data not shown). The most conspicuous changes were found in the pyramidal 


\begin{tabular}{lllll}
\hline Table 1. & Neuropathological findings in the $\mathbf{C A 3 / 2}$ region & & \\
$\begin{array}{llll}\text { Monkey } \\
\text { (implant) }\end{array}$ & $\begin{array}{l}\text { Shrinkage and } \\
\text { condensation of soma } \\
\text { and nucleus }\end{array}$ & $\begin{array}{l}\text { Percentage of } \\
\text { neurons with } \\
\text { such traits }\end{array}$ & Dendritic atrophy & $\begin{array}{c}\text { Cell layer } \\
\text { irregularity }\end{array}$ \\
\hline $\begin{array}{l}\text { Cholesterol } \\
1\end{array}$ & none & 0 & none & \\
2 & none & 0 & none & some \\
3 & mild & 4 & none & some \\
4 & none & 0 & none & none \\
Cortisol & mild & & & none \\
1 & moderate & 8 & mild & mild \\
2 & mild & 20 & moderate & mild \\
3 & marked & 2 & mild & mild \\
4 & 44 & marked & moderate
\end{tabular}

In all categories, mild pathology is defined as $1-10 \%$ of neurons involved; moderate as $11-20 \%$ involved; and marked as more than $20 \%$ of neurons involved.

neurons in the CA3 and CA2 regions; there was preferential damage in hippocampi implanted with cortisol-secreting pellets, as compared with cholesterol control sides (Table 1). In cholesterol-treated hippocampi, the CA3 region consisted of regularly arranged pyramidal neurons and their well-developed dendrites associated with the dense zone of the mossy fiber endings in the zona lucidum (Fig. 2a). All such neurons had a large round nucleus with a distinct nucleolus; perikaryonic were stretched and formed a broad dendritic stem with a rich arborization of dendritic branches in the zona lucidum (Fig. 2b). In contrast, in all cortisol-treated hippocampi, there were neurons with abnormal shrinkage or elongation of the soma associated with dark staining and condensation of both the nucleus and cytoplasm (including the dendritic stem; Fig. 2, c, d). The zonal arrangement showed some irregularity in the cortisol-treated hippocampi, and some areas of the CA3 region consisted of denscly populated degenerative ncurons whose slender dendritic branches showed darkly stained dendroplasma (Fig. 2d).

In silver stained sections of the CA3 region from cholesteroltreated hippocampi, the mossy fibers and their endings had a fibrillar appearance with fine dots (Fig. 3a). In contrast, in cortisol-treated hippocampi, the mossy fibers and their endings showed a coarse, button-like swelling along the dendritic stems (Fig. 3b).

On a quantitative level of analysis, there were no differences in numbers of neurons in cortisol- and cholesterol-implanted hippocampi (Table 2).

No gliosis or neuronophagia was observed in any hippocampi. There was no damage noted in the hippocampus outside the $\mathrm{CA} 3 / \mathrm{CA} 2$ region, nor in the cortex.

\section{Discussion}

The present study examined whether 1 year of GC exposure damages the primate hippocampus, and this appears to be the case. Cell layer irregularity occurred in half of the cholesteroltreated hippocampi, perhaps a neuropathologic response to the pellet itself; no further signs of degeneration were noted. In contrast, all cortisol-treated hippocampi had cell layer irregularity. Moreover, all cortisol-treated hippocampi showed soma shrinkage, dark staining, condensation, and/or dendritic atrophy. Such degeneration was severe in some cases. The cortisolassociated damage occurred only in $\mathrm{CA} 3$ and $\mathrm{CA} 2$ regions. These were not the hippocampal regions that were necessarily closest to the pcllets; instcad, this represents an intrinsic vulncrability of these neurons to the damaging effects of the cortisol.

These data agree with the growing literature concerning GCor stress-induced hippocampal degeneration in the rat. In that species, excessive exposure to GCs can accelerate hippocampal neuron loss during aging. Moreover, GCs can increase the extent of hippocampal damage following a number of neurological insults. Some recent studies have cast light on the cellular mechanisms by which GCs endanger the hippocampus (reviewed in Sapolsky, 1990).

These data also agree with a small literature concerning the effects of GCs or stress upon the primate hippocampus. In one study, $133 \mathrm{~d}$ fetal rhesus monkeys were exposed to high $(5 \mathrm{mg} /$ $\mathrm{kg}$ mother's body weight) concentrations of the synthetic GC, dexamethasone. Near term, considerable hippocampal damage was observed, including loss of pyramidal neurons, swelling of dendritic branches and of axon terminals, perikaryal atrophy and dispersion of vesicles, mitochondria and neurotubules (Uno et al., 1983). In one study of adult primates, preferential hippocampal degeneration was observed in vervet monkeys who died of a syndrome of sustained social stress (Uno et al., 1989b). As noted, while animals almost certainly hypersecreted GCs during the stressful period (as evidenced by hyperplastic adrenals), it was not known whether GCs were the proximal damaging agents.

The present data also agree with the existing rodent and primate literature in terms of the neuroanatomy of hippocampal vulnerability to GCs. Cortisol-treated hippocampi had damage limited to the CA3 and CA2 regions. In the aging rodent, hippocampal neuron loss is most dramatic in the $\mathrm{CA} 3 / \mathrm{CA} 2$ region, while CA 1 , subiculum, and dentate gyrus are relatively resistant (Coleman and Flood, 1987). Moreover, in the young adult rat, the endangering effects of prolonged GC exposure, and the pro-

Table 2. Numbers of neurons in the hippocampal region
\begin{tabular}{lllll} 
Implant & Dentate & CA4 & CA3 & CAl \\
\hline Cholesterol & $907 \pm 85$ & $401 \pm 19$ & $226 \pm 26$ & $288 \pm 34$ \\
Cortisol & $991 \pm 71$ & $390 \pm 10$ & $250 \pm 16$ & $291 \pm 19$
\end{tabular}

No statistical differences were noted between the 2 treatment groups. Numbers of neurons are given per square millimeter. Degenerating neurons are included in counts. 


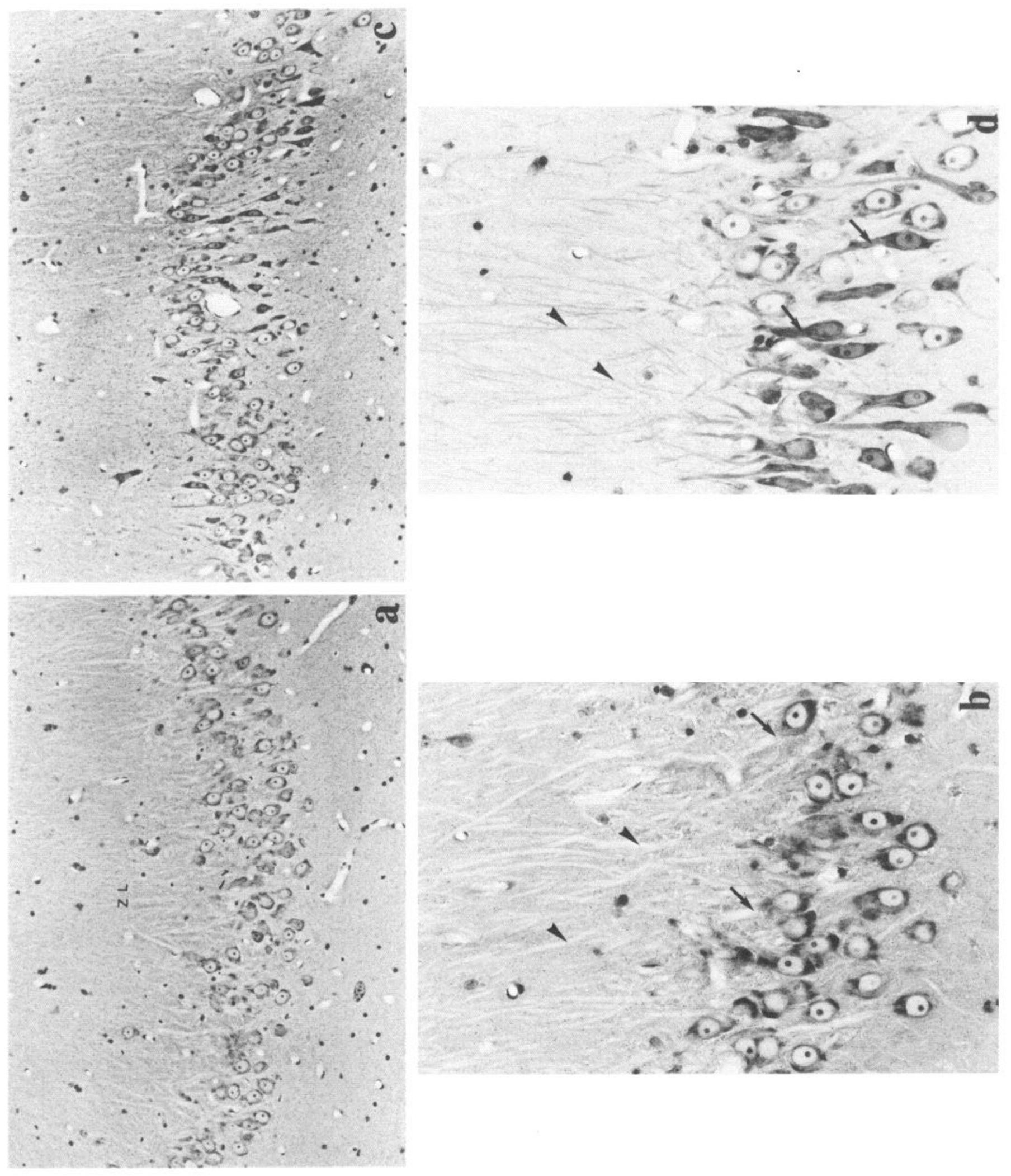



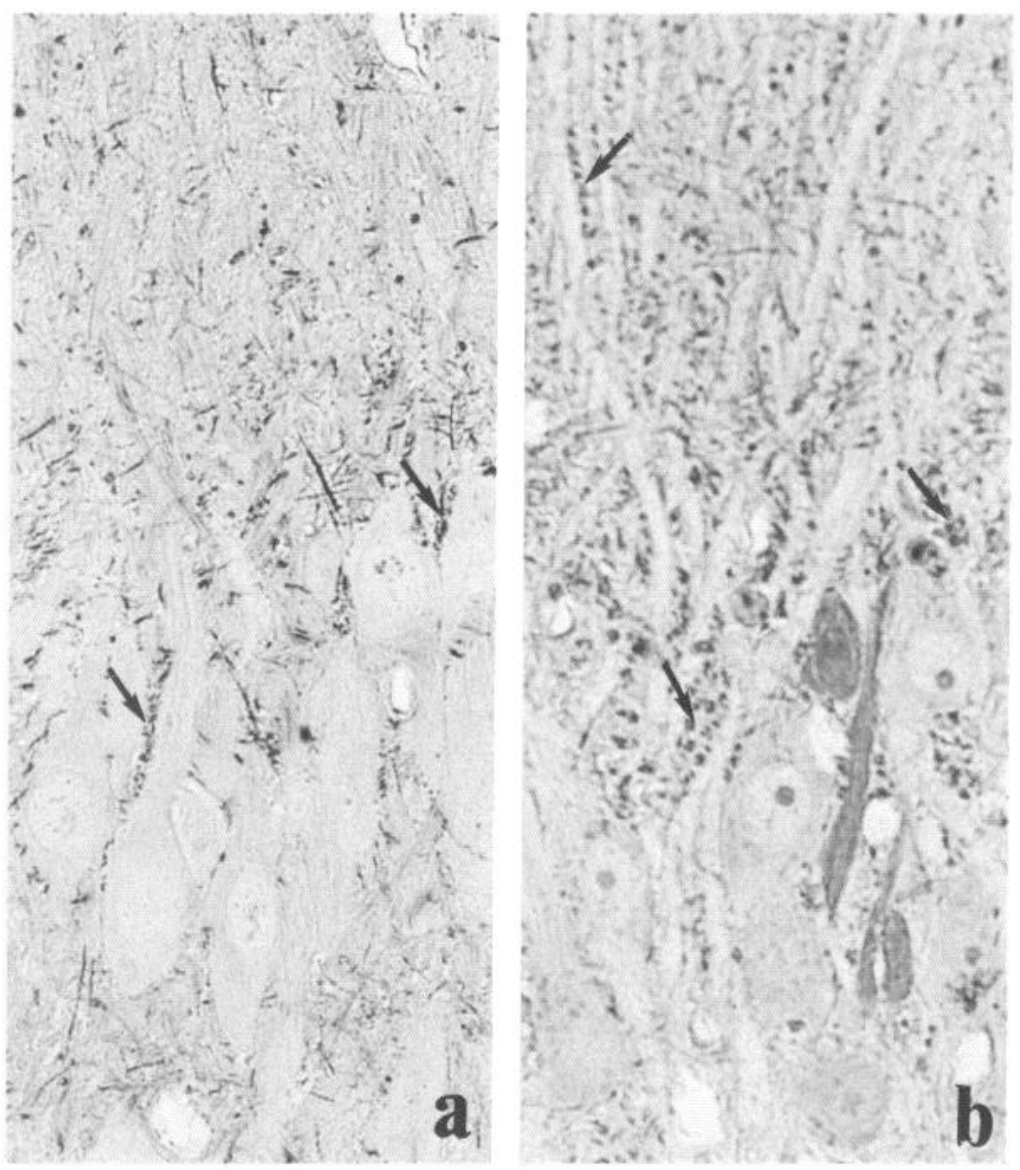

Figure 3. $a$, Silver-impregnation of plastic sections ( $3 \mu$ thick) of $\mathrm{CA} 3$ pyramidal neurons showing the fine fibrillar and dot-like appearance of the mossy fibers and their terminals around the soma and dendritic stems (arrows) in the cholesterol-implanted side, $\times 400$. $b$, In the cortisol-implanted side the terminal endings around the soma and dendrites of normal pyramidal neurons show a coarse button-like swelling (arrows), $\times 400$.

tective effects of behavioral or surgical reductions in GC exposure are both most dramatic in the CA3 region and least effective in dentate gyrus and CA1 (Landfield et al., 1981; Sapolsky et al., 1985; Meaney et al., 1988). This preferential vulnerability was also noted in the chronically-stressed vervet monkeys (Uno et al., 1989b). Damage was most severe in the CA3 region, with lesser amounts in CA1 and CA4, and no damage in dentate gyrus, thalamus, hypothalamus, caudate, or pontine nuclei. Furthermore, in the study of dexamethasone neurotoxicity in the fetal rhesus monkey (Uno et al., 1983), while damage occurred throughout the hippocampus, the most dramatic degeneration of axodendritic synaptic terminals occurred in the CA3 region.

The subcellular pattern of degeneration in such chronicallystressed adult monkeys (Uno et al., 1989b) was also similar to the "simple atrophy" seen in the present case. Such neurons had darkly stained condensation and shrinkage as well as dendritic atrophy; nuclei were shrunken and contained condensed chromatin. Ultrastructurally, such neurons contained less mi- tochondria, Nissl bodies, and had dilated endoplasmic reticulum and vacuoles (Uno et al., 1989b). In both studies, there was minimal glial reactivity. No invasive gliosis occurred in the present case, and in the stress study, only generalized oligodendrocytic swelling occurred, which is most probably a nonspecific artifact of either agonal state or postmortem autolysis (Penfield and Cone, 1926; Brierley and Graham, 1984). Similarly, the prenatal neurotoxicity of dexamethasone in the rhesus hippocampus (Uno et al., 1983) was not associated with glial reactivity.

The most striking difference among these studies is that no neuron loss occurred in the present study. In contrast, the aging rodent hippocampus sustains neuron loss as well as degeneration of surviving neurons, while the manipulations of cumulative GC exposure alter numbers of neurons. In addition, the hippocampi of the chronically stressed vervet monkeys and of the rhesus exposed prenatally to dexamethasone had neuron loss, as well as degeneration of remaining neurons. However, the hippocampi in the present study appear to be in a transitional

Figure 2. $a$, The pyramidal neurons in the CA3 region of the cholesterol implanted side, showing the regular zonal arrangement of neurons and dense dendritic arborization in the zona lucidum $(Z L), \times 100 . b$. High power magnification of the CA3 region of the cholesterol implanted side, showing the homogenous appearance of pyramidal neurons possessing a large round and clear nucleus containing a distinct nucleolus. Broad dendritic stems (arrows) stretch from perikaryonic soma containing rich Nissl's bodies and dendritic branches form rich arborization in the zona lucidum (arrowhead), $\times 250$. $c$, The CA3 region of the cortisol implanted side, showing shrinkage of neurons associated with darkly stained condensation of the soma coexisting with normal neurons and irregularity of the zonal arrangement, $\times 100$. $d$, High power magnification of CA3 neurons of the cortisol-implanted side, showing spindle-shaped elongation or shrinkage of the soma associated with darkly stained condensed cytoplasma, nucleus, and dendritic stem and branches (arrows). Some neurons retain a normal appearance (arrowheads), $\times 250$. 
state where damage is not yet severe enough to have caused neuron loss in addition to the qualitative signs of damage. These pathologic findings suggest that there was less exposure to GCs than in the previous reports. It was not possible to determine the level of GCs (nor cholesterol) to which the hippocampi were exposed in this study. (In addition, it was not possible to determine if the rate of release for cortisol and cholesterol were equal and/or constant, although the pellets are designed to allow fairly constant and equal rates of release for all steroids.) $\mathrm{Be}$ cause the secretion by the pellets was local, their outflow would not be reflected in changes in circulating GC concentrations, and thus it is not possible to state whether the overexposure equaled that which would occur with sustained physiological stress, with a pathological state such as Cushings syndrome, or with exogenous GC administration of a certain dose. Clarifying the physiological and/or pharmacological relevance of this observation awaits further study.

GC neurotoxicity in the hippocampus might be of clinical importance. In the rat, GC administration following hypoxiaischemia augments hippocampal damage (Sapolsky and Pulsinelli, 1985). Were the same to occur in humans, the current use of GCs to control post-stroke edema would be strongly contraindicated. In the rat, adrenalectomy following hypoxia-ischemia or excitotoxic seizures decreases hippocampal damage (Sapolsky, 1985, 1986; Sapolsky and Pulsinelli, 1985; Theoret et al., 1985). GC secretion is considerable following these stressful events (Stein and Sapolsky, 1988), and is apparently large enough to exacerbate hippocampal damage. Thus, what is viewed as "typical" hippocampal damage after these insults may be typical damage worsened by the coincident stress-response. Were that applicable to humans, strategies for pharmacologically reducing GC secretion at such times could be protective, as has been shown with the rat (Stein and Sapolsky, 1988; Morse and Davis, 1989). Finally, sustained GC overexposure in the rat, even in the absence of neurological insults, eventually damages the hippocampus. Were such to occur in humans, sustained stress or sustained GC administration (as is done for a vast number of clinical disorders) could have neuropathologic consequences. The present data, we feel, make GC-induced hippocampal damage in the human a more plausible scenario. As an obvious caveat, however, direct application of GCs to the brain is different from systemic application or endogenous release, and the present findings should be interpreted with caution.

\section{References}

Aus der Muhlen K, Ockenfels H (1969) Morphologische veranderungen im deincephalon und telencephalon nach storngen des regelkreises adenohypophyse-nebennierenrinde III. Ergebnisse beim meerschweinchen nach verabreichung von cortison und hydrocortison. $\mathrm{Z}$ Zellforsch 93:126-138.

Brierley J, Graham D (1984) Hypoxia and vascular disorders of the central nervous system. In: Greenfield's neuropathology, 4th ed (Adams J, Corsellis J, Duchen L, eds), pp 125-207. New York: Wiley.

Coleman P, Flood D (1987) Neuron numbers and dendritic extent in normal aging and Alzheimer's disease. Neurobiol Aging 8:521-546.

Kerr D, Applegate M, Campbell L, Goliszek A, Brodish A, Landfield P (1986) Chronic stress-induced acceleration of age-related hippocampal neurophysiological changes. Soc Neurosci Abstr 12:274.

Koide T, Wieloch T, Siesjo B (1986) Chronic dexamethasone pretreatment aggravates ischemic neuronal necrosis. J Cerebr Blood Flow Metab 6:395-403.

Landfield P, Baskin R, Pitler T (1981) Brain aging correlates: retardation by hormonal-pharmacological treatments. Science 214:581584.

McEwen B, de Kloet E, Rostene W (1986) Adrenal steroid receptors and actions in the nervous system. Phys Rev 66:1121-1189.

Meaney M, Aitken D, Bhatnager S, van Berkel C, Sapolsky R (1988) Effect of neonatal handling on age-related impairments associated with the hippocampus. Science 239:766-769.

Morse J, Davis J (1989) Chemical adrenalectomy protects hippocampal cells following ischemia. Soc Neurosci Abstr 149:4.

Penfield W, Cone W (1926) Acute swelling of oligodendroglia. A specific type of neuroglia change. Arch Neurol Psychiatry 16:131-153.

Rakic P, Nowakowski R (1981) The time of origin of neurons in the hippocampal region of the rhesus monkey. J Comp Neurol 196:99128.

Rebert C (1988) Neurophysiological bases of event-related potentials. Final report, AFOSR Contract F49620-82-K-0016. Menlo Park, CA: SRI International.

Sapolsky R (1985) A possible mechanism for glucocorticoid toxicity in the hippocampus: increased vulnerability of neurons to metabolic insults. J Neurosci 5:1228-1232.

Sapolsky R (1986) Glucocorticoid toxicity in the hippocampus: reversal by supplementation with brain fuels. J Neurosci 6:2240-2247.

Sapolsky R (1990) Glucocorticoids, hippocampal damage and the glutamatergic synapse. Prog Brain Res (in press).

Sapolsky R, Pulsinelli W (1985) Glucocorticoids potentiate ischemic injury to neurons: therapeutic implications. Science 229:1397-1400.

Sapolsky R, Krey L, McEwen B (1985) Prolonged glucocorticoid exposure reduces hippocampal neuron number: implications for aging. J Neurosci 5:1121-1227.

Stein B, Sapolsky R (1988) Chemical adrenalectomy reduces hippocampal damage induced by kainic acid. Brain Res 473:175-181.

Sumi S, Truog W, Kessler D (1984) Maternal corticosteroid therapy and the fetal brain in experimental hyalin membrane disease. Pediatr Res 18:440-444.

Theoret Y, Caldwell-Kenkel J, Krigman M (1985) The role of neuronal metabolic insult in organometal neurotoxicity. Tuxicologist (abstr) 6 : 491.

Uno H, Thieme C, Kemnitz J, Farell P (1983) Effect of dexamethasone on the cerebral cortical development of the rhesus monkey. Soc Neurosci Abstr 320:3.

Uno H, Schroeder B, Alsum P, Takahashi L, Kalin N (1989a) The effect of prenatal stress on the hippocampus in rats. Soc Neurosci Abstr 110:6.

Uno H, Tarara R, Else I, Suleman M, Sapolsky R (1989b) Hippocampal damage associated with prolonged and fatal stress in primates. J Neurosci 9:1705-1711.

Uno H, Lohmiller L, Thieme C, Kemnitz J, Engle M, Roecker E, Farrell $P$ (1990) Brain damage induced by prenatal exposure to dexamethasone in fetal rhesus macaques. I. Hippocampus. Dev Brain Res (in press). 